\title{
An Integration-Type High-Speed Analog-to-Digital Converter
}

\author{
KAZUYUKI KONDO, MEMBER, IEEE, AND KENZO WATANABE, SENIOR MEMBER, IEEE
}

\begin{abstract}
An analog-to-digital (A/D) converter consisting of a switched-capacitor integrator, comparator, and control circuit is presented. The conversion process consists of voltage-to-frequency (V/F) conversion to determine the upper $M$ bits of an $N$-bit representation of an analog input voltage and the subsequent voltage-to-time (V/T) conversion to determine the remaining lower $N-M$ bits. The total clock cycle required for $N$-bit resolution is $2^{M}+2^{N-M}$ at most. The circuits for the $V / F$ and $V / T$ conversion share most of the components and thus the converter can be implemented with the minimum component count. Error analysis shows that a conversion accuracy higher than 12 bits can be expected from its CMOS monolithic realization. Prototype converters built using discrete components have confirmed the principles of operation.
\end{abstract}

\section{INTRODUCTION}

$\mathrm{D}^{2}$ UAL-SLOPE and charge-balancing analog-to-digital (A/D) converters are two typical integration-type converters commonly used in the instrumentation and measurement field [1]. Though different in the principle, i.e., the former is based on a voltage-to-time (V/T) conversion while the latter on a voltage-to-frequency $(\mathrm{V} / \mathrm{F})$ conversion, their conversion processes are both insensitive to component variations and also immune to powerline noise if the integration time is made equal to the period of the line frequency [2]-[4]. By virtue of these distinctive properties, excellent linearity and a resolution higher than 14 bits can be easily obtained. They require, however, at least $2^{N}$ clock cycles for $N$-bit resolution, and thus the conversion speed is too slow for many applications. To increase the applicability, an improvement in speed is highly desirable.

A well-acknowledged method for increasing the conversion rate of an integration-type $A / D$ converter is the use of a multislope technique [5]. For 4-digit resolution, a conversion rate as high as $1600 \mathrm{samples} / \mathrm{s}$ is possible with this method [6], but a complicated circuit design is inevitable because of the precisely weighted current sources required for the high-speed time measurement.

The improvement in speed is also possible by performing a $\mathrm{V} / \mathrm{F}$ conversion during the integration period of a dual-slope A/D converter. Based on this idea, an integrating-type high-speed A/D converter has been devel-

Manuscript received April 23, 1989; revised August 23, 1989.

K. Kondo is with the Department of Electrical Engineering, Suzuka College of Technology, Shiroko, Suzuka 510-02, Japan.

$\mathrm{K}$. Watanabe is with the Research Institute of Electronics, Shizuoka University, Hamamatsu 432, Japan.

IEEE Log Number 8931835. oped. The V/F conversion determines the upper $M$ bits of an $N$-bit binary representation of an analog voltage. The remaining lower $N-M$ bits are determined by the subsequent $\mathrm{V} / \mathrm{T}$ conversion. The total clock cycle required for $N$-bit resolution is thus $2^{M}+2^{N-M}$ at most. The $\mathrm{V} / \mathrm{F}$ and $\mathrm{V} / \mathrm{T}$ converters involved share most of the components and thus the speed improvement is accomplished with a minimum component count.

\section{Circuit Description}

Fig. 1 shows the circuit diagram of the integration-type high-speed A/D converter. Here, $V_{x}$ is an analog voltage to be converted into a binary number with reference to the voltage $V_{r}$. The whole circuit is divided into three main blocks; the switched-capacitor integrator, the comparator, and the control circuit including $M$ - and $K$-bit counters. The control circuit generates the digital signals to manage the converter operation. The timing is shown in Fig. 2. $\phi$ and $\bar{\phi}$ are the nonoverlapping two-phase clocks. $\phi_{R}$ is the reset pulse in synchronism with the $\phi$ clock. After resetting the integrator and the counters, the reset pulse initiates the A/D conversion. The state signal divides the conversion cycle into the two states; the $\mathrm{V} / \mathrm{F}$ conversion state $T_{\mathrm{VF}}$ during which the upper $M$ bits are determined by the charge-balancing technique, and the $\mathrm{V} /$ $\mathrm{T}$ conversion state $T_{\mathrm{VT}}$ during which the lower $K$ bits ( $K$ $=N-M$ ) are determined by the single-slope technique. The operation in each state for unipolar and for bipolar conversion will be described in detail next.

\section{A. Unipolar Conversion}

1) Operation in the $T_{\mathrm{VF}}$ State: In this state, switches $S_{1}$ and $S_{2}$ are kept "off." The circuit configuration and the control signal for each switch applicable in this state are shown in Fig. 3. Op-amp $A_{1}$ and capacitors $C_{1}, C_{2}$, and $C_{3}$ form the offset-free, parasitic- and gain-insensitive integrator [7], [8]. In the $\phi=" 1$ " phase, $C_{1}$ is charged, via $S_{4}, S_{8}$, and $C_{3}$, to an input voltage $V_{x}$. For the moment, $V_{x}$ is assumed to be positive. In the subsequent $\bar{\phi}$ $=$ " 1 " " phase, the charge stored in $C_{1}$ is transferred into $C_{2}$ via $S_{5}$ and $S_{6}$. The output voltage $V_{o}$ of the integrator thus increases by $C_{1} V_{x} / C_{2}$ in every $\bar{\phi}$ clock. This charge accumulation continues until the integrator output $V_{o}$ becomes positive and the comparator output (CMP) turns " 1 ." When this happens, the $M$-bit counter is incremented and switch $S_{3}$ is activated, instead of $S_{5}$, in the 


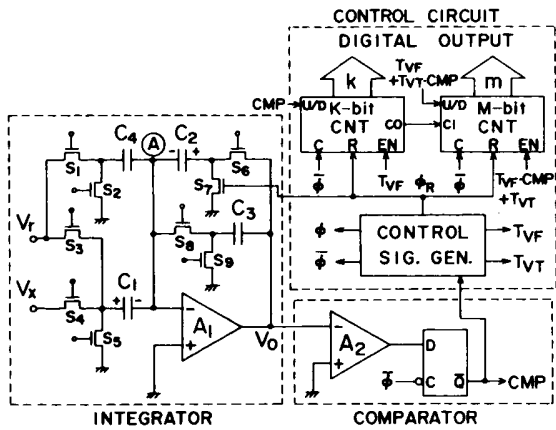

Fig. 1. The circuit diagram of the integration-type high-speed A/D converter.

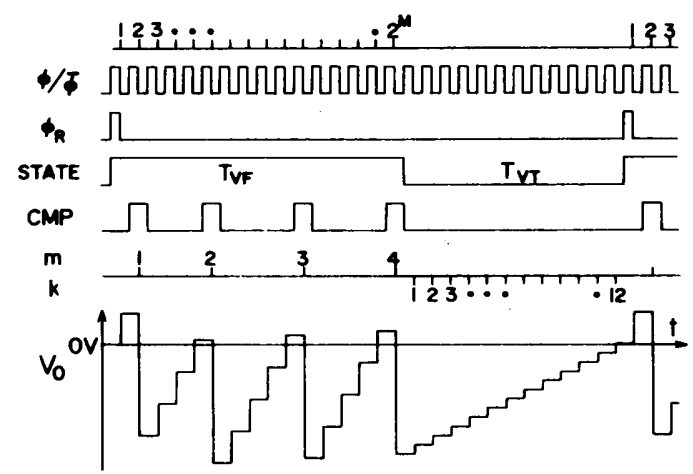

Fig. 2. The timing diagram of digital control signals and the output waveform of op-amp $A_{1}$.

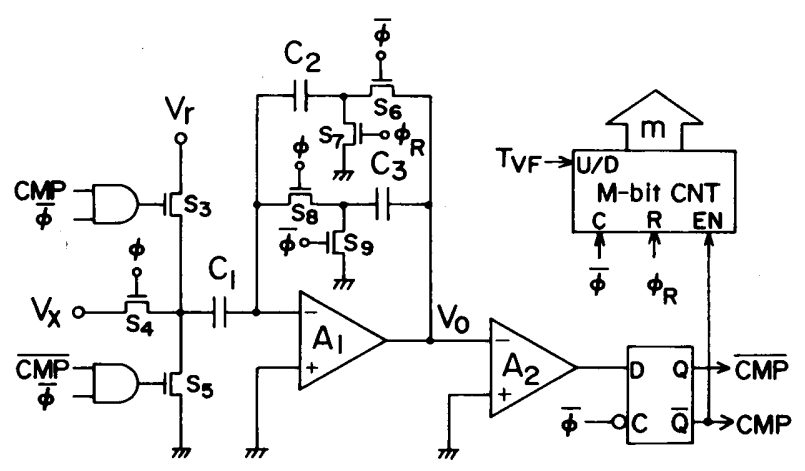

Fig. 3. The converter configuration in the $T_{\mathrm{VF}}$ state (unipolar conversion).

subsequent $\bar{\phi}=$ " 1 " phase. Then, the charge $C_{1}\left(V_{r}-\right.$ $V_{x}$ ) is extracted from $C_{2}$ and the integrator output becomes negative again, to resume the charge accumulation. This process of charge accumulation and extraction is repeated for $2^{M}$ clock cycles. Output voltage waveforms of the integrator and the comparator are also illustrated in Fig. 2.

Assume now that the charge extraction occurred $m$ times during this state. Then, the total reference charge extracted from $C_{2}$ is $m C_{1} V_{r}$, while the total signal charge accumulated into $C_{2}$ is $2^{M} C_{1} V_{x}$. The charge $Q\left(t_{M}\right)$ residing in $C_{2}$ at the end of this state is then

$$
Q\left(t_{M}\right)=C_{2} V_{o}\left(t_{M}\right)=C_{1}\left(2^{M} \mathrm{~V}_{x}-\mathrm{mV}_{r}\right) .
$$

Since $\left|Q\left(t_{M}\right)\right|<C_{1} V_{r}$, we have

$$
\begin{aligned}
\mid V_{x} & -\left(m_{1} 2^{-1}+m_{2} 2^{-2}+\cdots+m_{M} 2^{-M}\right) V_{r} \mid \\
& <2^{-M} V_{r}
\end{aligned}
$$

where

$$
\sum_{i=1}^{M} m_{i} 2^{M-i}=m
$$

Equation (2) indicates that $m_{i}(i=1,2, \cdots, M)$, the content of the $M$-bit counter, is an $M$-bit binary representation of $V_{x}$. To increase the resolution further, the output voltage $V_{o}\left(t_{M}\right)$ of the integrator is quantized by means of $\mathrm{V} / \mathrm{T}$ conversion in the subsequent $T_{\mathrm{VT}}$ state.

2) Operation in the $T_{\mathrm{VT}}$ State: In this state, switches $S_{4}$ and $S_{7}$ are kept "off." The circuit configuration and the control signal driving each switch are shown in Fig. 4. Op-amp $A_{1}$ and capacitors $C_{1}, C_{2}, C_{3}$, and $C_{4}$ now form a differential integrator. The comparator, consisting of op$\operatorname{amp} A_{2}$ and $D$ flip-flop, monitors the polarity of the integrator output to control the operation of the integrator and the $(M+K)$-bit up/down counter. If the output voltage $V_{o}\left(t_{M}\right)$ of the integrator at the end of the preceding $T_{\mathrm{VF}}$ state is positive, i.e., CMP $=$ " 1 ," then the integrator deposits the charge $C_{1} V_{r}$ onto $C_{2}$, in every $\bar{\phi}=$ " 1 " phase, while drawing the charge $C_{4} V_{r}$ from $C_{2}$ concurrently. The $(M+K)$-bit counter then operates as an up counter. If CMP $=$ " 0 ," on the other hand, then the charge $C_{4} V_{r}$ is deposited, in every $\bar{\phi}=$ " 1 " " phase, while the charge $C_{1} V_{r}$ is drawn concurrently. The counter then operates as a down counter.

Assume now that capacitors $C_{1}$ and $C_{4}$ consist of $2^{K}$ and $2^{K}+1$ unit capacitors, respectively, connected in parallel; $C_{1}=2^{K} C_{u}$ and $C_{4}=\left(2^{K}+1\right) C_{u}$, where $C_{u}$ denotes the unit capacitance. Then the net charge $Q_{n}$ :

$$
Q_{n}=C_{u} V_{r}
$$

is deposited onto or drawn from $C_{2}$ in every $\bar{\phi}=$ " 1 ", phase, depending on the polarity of $V_{o}\left(t_{M}\right)$. This process of charge deposit or withdrawal is repeated $k$ times, as illustrated in Fig. 2, until the total quantum charge $k C_{u} V_{r}$ counterbalances $Q\left(t_{M}\right)$. Thus we have

$$
\begin{aligned}
Q\left(t_{M}\right)= & \operatorname{sgn}\left[V_{o}\left(t_{M}\right)\right] k C_{u} V_{r} \\
= & \operatorname{sgn}\left[V_{o}\left(t_{M}\right)\right]\left(k_{1} 2^{-1}+k_{2} 2^{-2}\right. \\
& \left.+\cdots+k_{K} 2^{-K}\right) C_{1} V_{r}
\end{aligned}
$$

where

$$
\operatorname{sgn}\left[V_{o}\left(t_{M}\right)\right]=\left\{\begin{aligned}
1, & \text { if } V_{o}\left(t_{M}\right) \geqq 0 \\
-1, & \text { otherwise }
\end{aligned}\right.
$$

and

$$
\sum_{i=1}^{K} k_{i} 2^{K-i}=k
$$

It follows, therefore, that $k_{i}(i=1,2, \cdots, K)$, the content of the $K$-bit counter, gives a $K$-bit representation of $Q\left(t_{M}\right)$. 


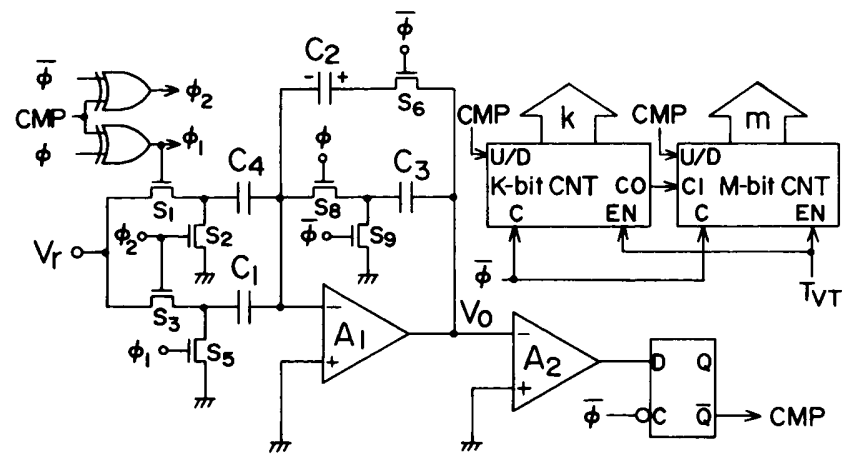

Fig. 4. The converter configuration in the $T_{\mathrm{VT}}$ state (unipolar conversion).

Combining (1) and (5), we can obtain an $N(=M+K)$ bit representation of an analog voltage $V_{x}$ :

$$
\begin{aligned}
V_{x}= & \left\{m_{1} 2^{-1}+m_{2} 2^{-2}+\cdots+m_{M} 2^{-M}\right. \\
& +\operatorname{sgn}\left[V_{o}\left(t_{M}\right)\right]\left(k_{1} 2^{-(M+1)}\right. \\
& \left.\left.+\cdots+k_{K} 2^{-(M+K)}\right)\right\} V_{r} .
\end{aligned}
$$

The total clock cycle required for the $N$-bit conversion is $2^{M}+2^{N-M}$ at most, which becomes minimum when $M$ $=N / 2$. A conventional integration-type converter, on the other hand, requires $2^{N}$ clock cycles at least. Therefore, the speed improvement as high as $2^{(N / 2)-1}$ is possible with the present architecture.

\section{B. Bipolar Conversion}

In the previous description of the converter operation, an input analog voltage $V_{x}$ was assumed to be positive. Bipolar conversion is also possible by slightly modifying the control signals in the $T_{\mathrm{VF}}$ state. The circuit diagram in the $T_{\mathrm{VF}}$ state for bipolar conversion is shown in Fig. 5. The timing diagram of the control signals and the output waveforms of the comparator and integrator when $M=K$ $=3$ are shown in Fig. 6. The analog circuitry is the same as that shown in Fig. 3, but the four-phase clocks ( $\phi_{a}$, $\phi_{b}, \phi_{c}$, and $\phi_{d}$ in Fig. 6) are required for driving each switch.

In $\phi_{a}$ and $\phi_{b}$ phases, op-amp $A_{1}$ operates as a noninverting integrator to accumulate the charge $C_{1} V_{x}$ onto $C_{2}$. Op-amp $A_{2}$ and $D$ flip-flop form the comparator to test the polarity of the integrator output $V_{o}$. If $V_{o}$ is positive, then the output of the comparator CMP is set to "1." Otherwise, CMP is set to " 0 ." In $\phi_{c}$ and $\phi_{d}$ phases, if CMP $=$ " 1 ," the reference charge $C_{1} V_{r}$ is extracted from $C_{2}$ and the $M$-bit counter is incremented. If CMP $=$ " 0 ," on the other hand, the reference charge $C_{1} V_{r}$ is accumulated in $C_{2}$. This process of charge accumulation and extraction is repeated for $2^{M}$ clock cycles of the four-phase clock.

Assume that the reference charge extraction occurred $m$ times in this state. Then the total extracted charge $Q_{\mathrm{ext}}$ is $m C_{1} V_{r}$, while the total charge $Q_{\text {dep }}$ deposited upon $C_{2}$ is $2^{M} C_{1} V_{x}+\left(2^{M}-m\right) C_{1} V_{r}$. The charge $Q\left(t_{M}\right)$ remaining in $C_{2}$ at the end of this state is then

$$
\begin{aligned}
Q\left(t_{M}\right) & =C_{1} V_{o}\left(t_{M}\right)=Q_{\mathrm{dep}}-Q_{\mathrm{ext}} \\
& =C_{1}\left\{2^{M}\left(V_{x}+V_{r}\right)-2 m V_{r}\right\} .
\end{aligned}
$$

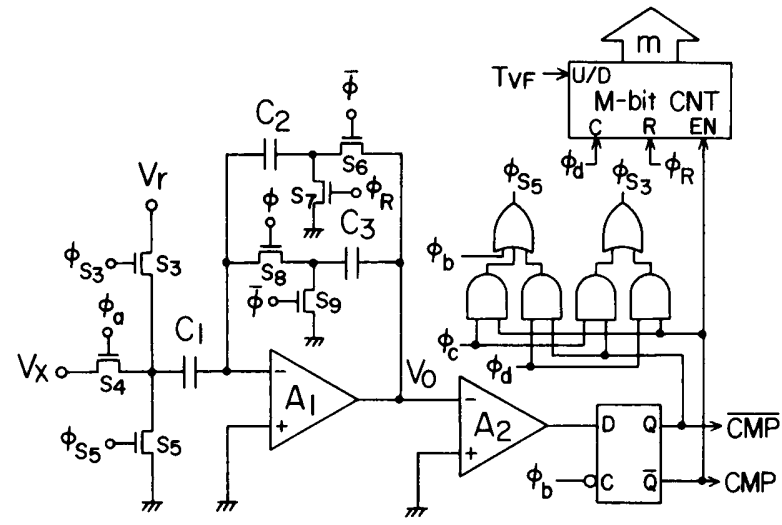

Fig. 5. The converter configuration in the $T_{\mathrm{VF}}$ state (bipolar conversion).

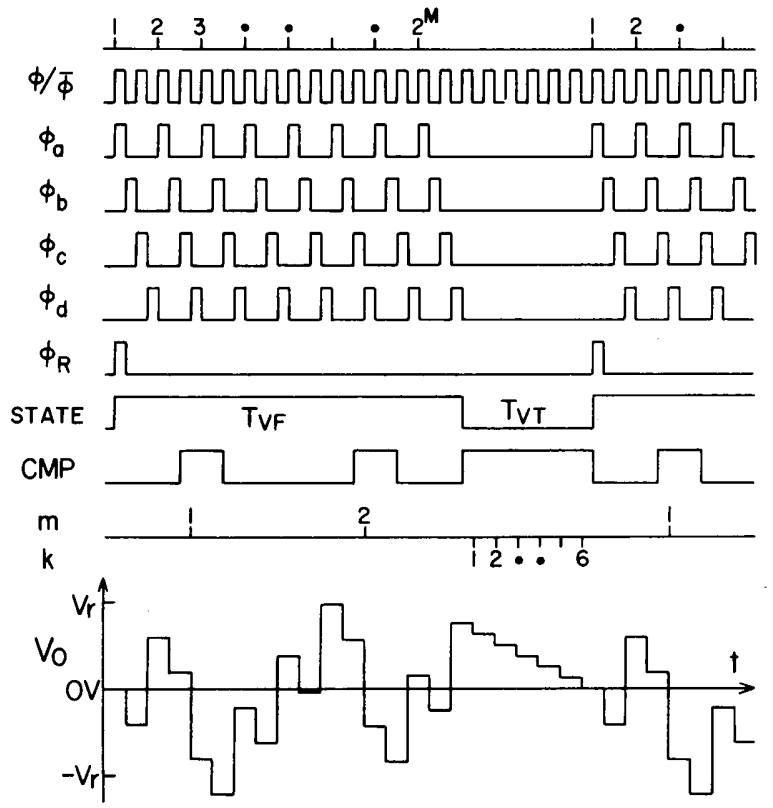

Fig. 6. The timing diagram of digital control signals and the output waveform of the integrator (bipolar conversion).

Since $\left|Q\left(t_{M}\right)\right|<C_{1} V_{r}$, we have

$$
\begin{aligned}
\mid V_{x} & -\left(m_{1} 2^{0}+m_{2} 2^{-1}+\cdots+m_{M} 2^{-M+1}-1\right) V_{r} \mid \\
& <2^{-M} V_{r}
\end{aligned}
$$

where $m_{i}(i=1,2, \cdots, M)$ is defined by (3).

The circuit configurations and operations in the $T_{\mathrm{VT}}$ state for bipolar conversion are the same as those for unipolar conversion, except that the charge $Q\left(t_{M}\right)$ can assume any value between $-C_{1} V_{r}$ and $C_{1} V_{r}$, and thus not a $K$-bit but $(K+1)$-bit up/down counter is required for quantizing the full scale value $2 C_{1} V_{r}$ using the quantum charge $C_{u} V_{r}$. Assume now again that the quantum charge is deposited onto or drawn from $C_{2} k$ times, depending on the polarity of $Q\left(t_{M}\right)$, as illustrated in Fig. 6, until the total quantum charge $k C_{u} V_{r}$ counterbalances $Q\left(t_{M}\right)$. Thus we have

$$
\begin{aligned}
Q\left(t_{M}\right)= & \operatorname{sgn}\left[V_{o}\left(t_{M}\right)\right] k C_{u} V_{r} \\
= & \operatorname{sgn}\left[V_{o}\left(t_{M}\right)\right]\left(k_{0} 2^{0}+k_{1} 2^{-1}+\cdots+k_{K} 2^{-K}\right) \\
& \cdot C_{1} V_{r}
\end{aligned}
$$


where

$$
\sum_{i=0}^{K} k_{i} 2^{K-i}=k
$$

Combining (9) and (11), we have

$$
\begin{aligned}
V_{x}= & \left\{\frac{m}{2^{M-1}}+\operatorname{sgn}\left[V_{o}\left(t_{M}\right)\right] \frac{k}{2^{K+M}}-1\right\} V_{r} \\
= & {\left[m_{1} 2^{0}+m_{2} 2^{-1}+\cdots+m_{M} 2^{-M+1}\right.} \\
& +\operatorname{sgn}\left[V_{o}\left(t_{M}\right)\right]\left\{k_{0} 2^{-M}+k_{1} 2^{-(M+1)}\right. \\
& \left.\left.+\cdots+k_{K} 2^{-(M+K)}\right\}-1\right] V_{r} .
\end{aligned}
$$

Therefore, $m$ and $k$ give an $(M+K+1)$-bit offset binary representation of $V_{x}$. The total number of clock cycles required for $(M+K+1)$-bit conversion is $2^{M+1}+2^{K}$ at most, since one cycle of the four-phase clock corresponds to two clock cycles and $Q\left(t_{M}\right)$ is quantized within $2^{K}$ clock cycles. Thus the conversion rate for a bipolar signal is the same as that for a unipolar signal.

\section{Conversion ACCuracy}

The switched-capacitor integrator is configured such that parasitic capacitances and the nonideal factors of opamp such as the offset voltage and the finite open-loop gain have no effect upon its operation. Therefore, the main error sources of the present A/D converter are clock feedthrough and the inaccuracy of the capacitance ratio between $C_{1}$ and $C_{4}$. Their effects upon the conversion accuracy will be estimated separately in this section. Only unipolar conversion is analyzed for simplicity, but the result holds also true for bipolar conversion.

\section{A. Clock Feedthrough}

Clock feedthrough accompanying each transition causes the offset charge to appear in $C_{2}$ in every cycle. The amount of the offset charge differs in the $T_{\mathrm{VF}}$ and $T_{\mathrm{VT}}$ states because the circuit configuration is different. For simplicity, however, it is assumed that the feedthrough charge $Q_{f}$ is injected into node (A) in Fig. 1 in every clock cycle. Then, the offset charge accumulated in $C_{2}$ during the whole conversion period amounts to $\left(2^{M}+2^{N-M}\right) Q_{f}$. If this offset charge is less than the quantum $C_{u} V_{r}$, then the $N$-bit conversion is accurate down to its LSB:

$$
2^{M}\left(2^{M}+2^{N-M}\right) \leqq \mathrm{C}_{1} \mathrm{~V}_{r} / \mathrm{Q}_{f} .
$$

This condition is most relaxed when $M=N / 2$. The signal-to-noise charge ratio $C_{1} V_{r} / Q_{f}$ as high as $1 \times 10^{4}$ can be obtained by accommodating the clock feedthrough cancellation scheme [9]. Then, a conversion accuracy higher than 12 bits can be expected.

\section{B. Capacitance Ratio Error}

The $\mathrm{V} / \mathrm{F}$ conversion in the $T_{\mathrm{VF}}$ state is independent of the capacitance ratio [4]. Thus it affects only the lower $K$ bits determined by the $\mathrm{V} / \mathrm{T}$ conversion. Assume now that unit capacitors composing $C_{1}$ and $C_{4}$ have the mismatch

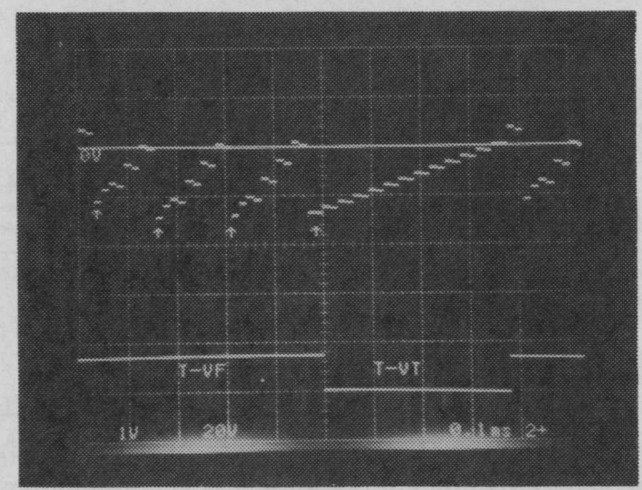

Fig. 7. The output voltage of op-amp $A_{1}$ (upper trace) and the state signal (lower trace) observed in the prototype 8-bit converter. Horizontal scale: $0.1 \mathrm{~ms} /$ div; vertical scale: $1 \mathrm{~V} / \operatorname{div}$ (upper trace), and $20 \mathrm{~V} /$ div (lower trace).

$\epsilon$. The maximum error in the quantum charge deposited onto or subtracted from $C_{2}$ during the $T_{\mathrm{VT}}$ state then amounts to $2^{K}\left(2^{(K / 2)+1}\right) \epsilon C_{u} V_{r}$. If this error is less than one quantum, then the $K$-bit conversion is accurate down to its LSB. This gives the accuracy condition

$$
2^{(K / 2)+1}\left(2^{K}-1\right) \epsilon<1 .
$$

The capacitance mismatch $\epsilon$ as small as 0.05 percent is attainable with presently available MOS technologies [10], [11]. Therefore, the accuracy of the V/T conversion is estimated to be 7 bits.

Summarizing the above discussions, we can conclude that the overall accuracy of the present converter is determined by clock feedthrough and a 12-bit resolution can be obtained by its CMOS monolithic realization. Choosing $M=K=N / 2$ gives the best compromise between the conversion accuracy and speed. If, however, the application allows the slower speed, then decreasing $K$ and increasing $M$ correspondingly would be beneficial in reducing the chip area, because capacitors $C_{1}$ and $C_{4}$ occupy most of the area.

\section{Experimental Results}

To confirm the principles of operation, prototype unipolar and bipolar converters were breadboarded using discrete components. Op-amps and switches used were LF347 and MC14016, respectively. Capacitors were $C_{1}$ $=2.63, C_{2}=2.81, C_{3}=11.2$, and $C_{4}=2.79 \mathrm{nF}$. The reference voltage was set to $2 \mathrm{~V}$.

Fig. 7 shows the integrator output and the state signal observed when the analog voltage $V_{x}=0.41 \mathrm{~V}$ was converted into 8 -bit ( $M=K=4$ ) binary number by the unipolar converter. In the $T_{\mathrm{VF}}$ state, the reference charge is extracted 4 times (pointed by white arrows) and thus $m=$ 4. In the $T_{\mathrm{VT}}$ state, the quantum charge is deposited 12 times, and thus $k=12$. Since $V_{o}\left(t_{M}\right)<0,(M+K)$-bit counter operates as a down counter. Therefore, the digital output is $01000000-00001100=00110100$. This corresponds to the analog voltage $V_{x}=(52 / 256) \times 2=$ $0.406 \mathrm{~V}$, agreeing well with the applied voltage. 


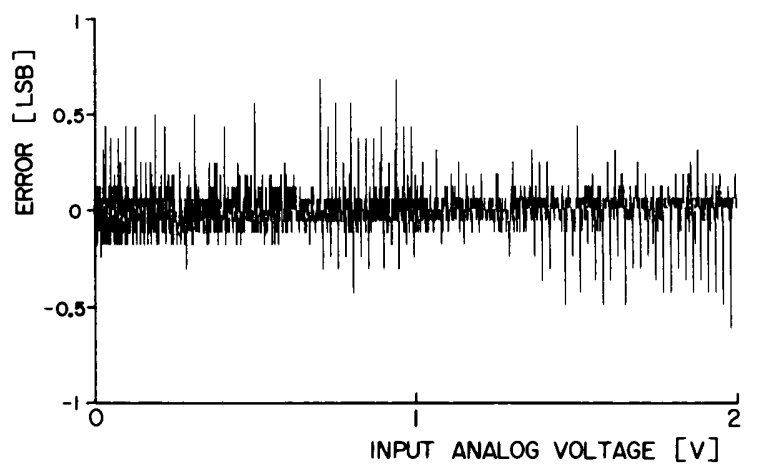

Fig. 8. The differential nonlinearity error of the 12-bit prototype A/D converter.

Fig. 8 shows the differential nonlinearity error of the 12-bit ( $M=8, K=4$ ) prototype unipolar converter measured by a static test. The maximum error is less than 0.7 LSB, and thus no missing code appears. A prototype bipolar converter has also confirmed the principles of operation and the accuracy estimate.

The clock rate used in these experiments was $30 \mathrm{kHz}$. In CMOS realization, the clock frequency higher than 10 $\mathrm{MHz}$ can be used, and thus a conversion rate compared favorably with that of a CMOS successive approximation A/D converter is attainable with the present architecture.

\section{Conclusions}

An integration-type high-speed A/D converter using a switched-capacitor circuit was presented. This converter features a small device-count integrable onto a small chip area, a high accuracy made possible by the parasitic and gain insensitive configuration, and a conversion rate com- parable to that of a successive approximation A/D converter. Therefore, it will find wide applicability in the instrumentation and measurement field.

\section{ACKNOWLEDGMENT}

K. Kondo would like to thank Prof. Y. Fukuoka, Department of Electrical Engineering, Suzuka College of Technology, for his encouragement.

\section{REFERENCES}

[1] B. M. Oliver and J. M. Cage, Ed., Electronic Measurement and Instrumentation. New York: McGraw-Hill, 1971, chap. 8.

[2] A. B. Grebene, Bipolar and MOS Analog Integrated Circuit Design. New York: Wiley 1984, chap. 15.

[3] D. J. Dooley, Ed., Data Conversion Integrated Circuits. New York: IEEE Press, 1980.

[4] H. Matsumoto and K. Watanabe, "A switched-capacitor charge-balancing analog-to-digital converter and its application to capacitance measurement," IEEE Trans. Instrum. Meas., vol. IM-36, pp. 873878, Dec. 1987.

[5] S. M. R. Taha, "Speed improvements for dual-slope A/D converters," IEEE Trans. Instrum. Meas., vol. IM-34, pp. 630-635, 1985.

[6] E. Yada, J. Honjo, and M. Mirose, "High-speed A/D converter with variable integration time," in IEEE Instrum. Meas. Tech. Conf. Rec., pp. 144-147, Mar. 1986.

[7] K. Haug, F. Maloberti, and G. C. Temes, "Switched-capacitor integrators with low finite gain sensitivity," Electron. Lett., vol. 21, pp. 1156-1157, Nov. 1985.

[8] K. Martin and A. S. Sedra, "Stray-insensitive switched-capacitor filters based on bilinear z-transform," Electron. Lett., vol. 15, pp. 365366, June 1979.

[9] K. Martin, "New clock feedthrough cancellation technique for analog MOS switched-capacitor circuits," Electron. Lett., vol. 18, pp. 3940, Jan. 1982.

[10] J. L. McCreary, "Matching properties, and voltage and temperature dependence of MOS capacitors," IEEE J. Solid-State Circuits, vol. SC-16, pp. 608-616, Dec. 1981.

[11] R. Gregorian and G. C. Temes, Analog MOS Integrated Circuits for Signal Processing. New York: Wiley, 1986, chap. 3. 